\title{
Experience Report: The Effectiveness of Paper Prototyping for Interactive Visualizations
}

\author{
Bastian Bansemir ${ }^{1}$, Franziska Hann $\beta^{2}$, Berit Lochner ${ }^{1}$, \\ Jan Wojdziak ${ }^{2}$, and Rainer Groh ${ }^{3}$ \\ ${ }^{1}$ BMW Group, München, Germany \\ \{bastian. bansemir, berit. lochner\} @bmw. de \\ ${ }^{2}$ Gesellschaft für Technische Visualistik mbH - GTV, Dresden Germany \\ \{franziska.hannss, jan.wojdziak\} @visuativ.com \\ ${ }^{3}$ Chair of Media Design, Technische Universität Dresden, Germany \\ rainer.grohatu-dresden.de
}

\begin{abstract}
This article describes the designing process of an interactive visualization at BMW. Paper prototyping is introduced as a part of the usercentered-design process and put into practice as a novel method for software development. During the development process paper prototypes have been used as a tool for communicating and testing within the interdisciplinary team of operational experts, visualization experts, designers and software developers. In conclusion the integration of paper prototyping benefits the design process positively. The interactive visualization meets the expectations of the user.
\end{abstract}

Keywords: paper prototyping, design research, interaction design, interface design, software development, interactive systems.

\section{$1 \quad$ Introduction}

Companies engage in speeding-up their software development cycles [1], due to harder competition, raising instability, increasing complexity or changing customer preferences [2, 3]. Indeed, notable successes have been achieved while moving away from traditional software development models, such as the waterfall model, to scrumbased or other agile models $[4,5]$.

This agile movement is impeded by the tendency to specialize employees, to intensify the workload for each employee, and to globally distribute employees [6]. Many issues evolve around agile models if employees do not work at the same or close location or even time zone or are not able to participate in daily meetings. However, realizing a high frequency in such situations, necessary for iterative software development, is often not handy or even impossible. Hence, agile models often fail to live-up to expectations companies often have [6].

At the intersection point between 'the dream of agility' and 'the daily reality' of a highly distributed workforce, paper prototyping is gaining momentum as one possible solution for solving this seeming contradiction. Paper prototyping may be understood 
as a cheap and quick tool to support the understanding between designers, developers, managers, engineers, customers and users of different design-stages during the development process [7].

Whilst extensive research has already studied various aspects of prototyping in the field of Human Computer Interaction in great detail [8-10] and has achieved compelling results, we focus in our case study on the use of paper prototyping. The major research question may be formulated as follows: How to use paper prototyping for user interface development of complex interactive systems? The study reports results from a case study conducted at and with BMW concerning the software development of a small-scale IT project. The main focus of software development concerned interface design of an interactive software project. Results indicate that paper prototyping may indeed close the gap between requirements of agile development and daily challenges of distributed workplaces.

The following sections shed light on first prototyping methods, second methodological proceeding applying paper prototyping and third outcomes of the paper prototyping process.

\section{Prototyping in the Design Process}

Within the process of interface design, prototyping is an essential tool for requirements elicitation, testing, communicating and decision-making. Prototypes represent a part or the complete user interface of the interactive system. They can be made with pen, paper and cardboard or by typing code. Prototypes may be segregated concerning their level of (i) completeness and (ii) fidelity.

First, prototyping methods are used to address special issues of the current state of the design and development process. In other words, it might either be necessary to give an impression of the whole system from the user's point of view or have an
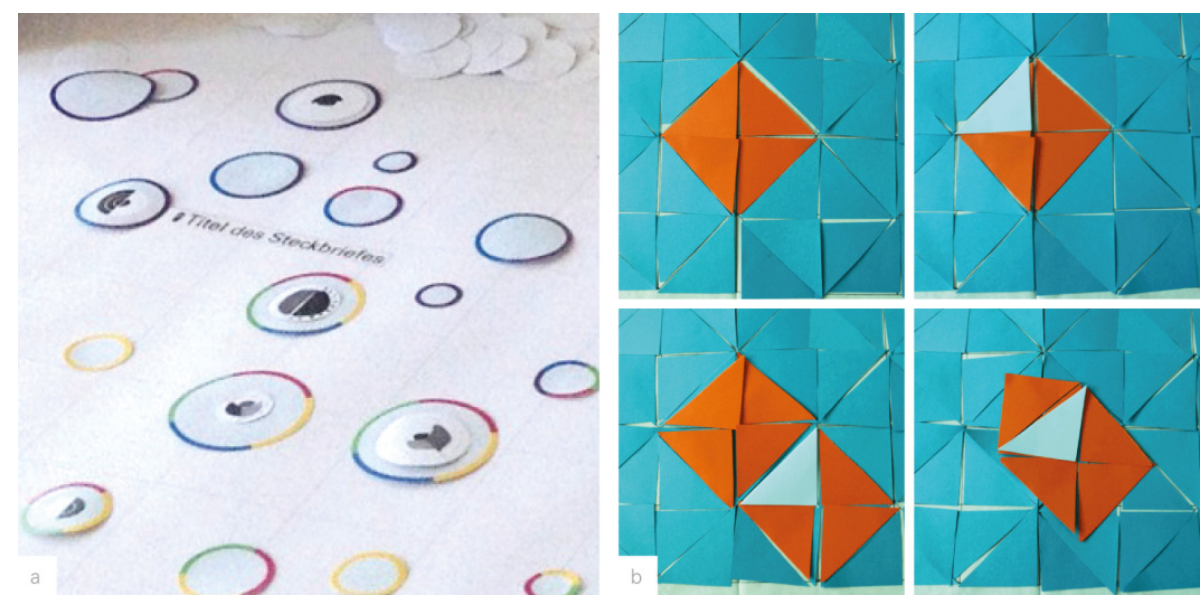

Fig. 1. Examples of Prototyping strategies: (a) horizontal prototype for a design decision process and (b) vertical prototyping as a stop motion video [11] 
in-depth discussion on a specific element of the system under development. Hence, concerning the completeness of a prototype, two different types are distinguished: If a prototype presents a big range (or even the full set) of features of the interface, it is called a horizontal prototype [8]. Fig. 1a introduces an example of a horizontal paper prototype for a design decision process of the complex interactive system that was developed during the second cycle of the prototyping process (see chapter 3 ). Contrary, to estimate the usability or feasibility of a specific feature of a given system a vertical prototype is used. Gaining a first impression of a feature's functionality is the main objective of vertical prototypes. Fig. $1 \mathrm{~b}$ shows a vertical prototype made out of paper. To achieve a better understanding of the functionality from a user's interaction perspective a stop-motion video is used to explicate the interaction aspect.

Second, due to their complexity prototypes may be characterized as low- or highfidelity prototypes. Low-fidelity prototypes are characterized by low development costs, are mainly used for evaluation of competing design concepts and are most useful as a communication device within the developer team [12]. Furthermore they help to make design decisions at an early stage of the design process (see fig. 2b). Low-fidelity prototypes allow teams to experiment with a big number of design alternatives (especially when compared to high-fidelity prototypes) [13]. On the other hand, high-fidelity prototypes show the look and feel close to the final application. Therefore, they often represent the full set of functions and inhabit full interactivity. These prototypes are often used for exploring issue related to navigation and testing [12]. Fig 2 a presents an interactive functional model of a product design process, i.e. a so-called work model.
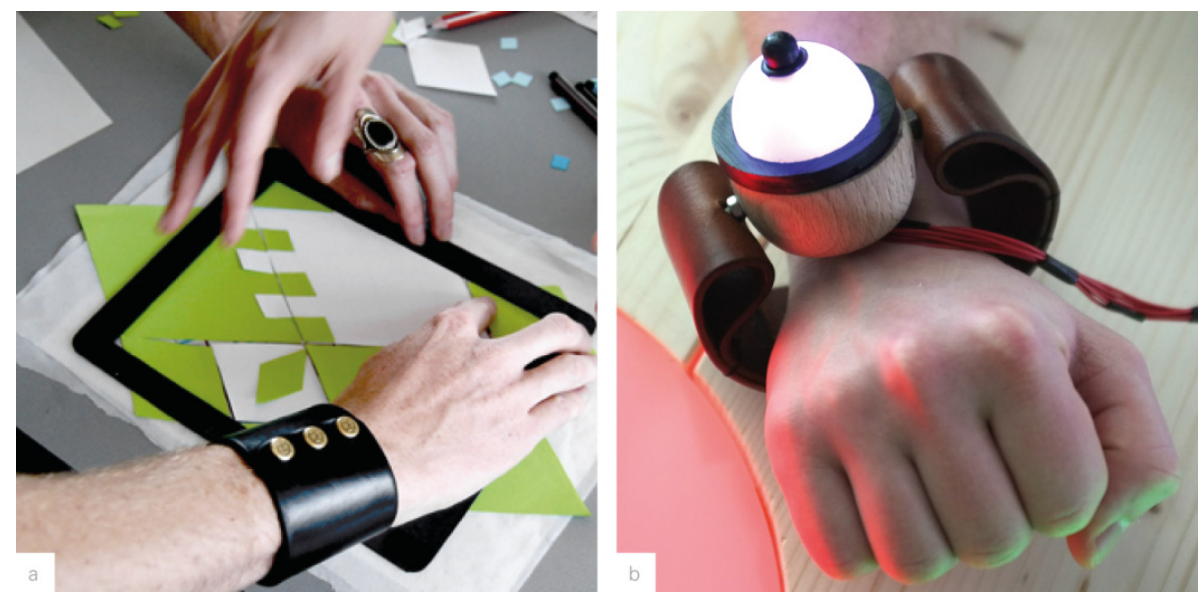

Fig. 2. Examples of prototyping methods regarding the level of fidelity: (a) low-fidelity prototypes for a decision making process concerning an interface structure and (b) high-fidelity prototyping as an interactive functional model of a product design process [11] 


\subsection{The Concept of Paper Prototyping}

Paper prototyping (also called offline prototyping) may result in artifacts, such as paper sketches, illustrated storyboard, cardboard mock-ups or stop motion videos [14]. Paper prototyping is mostly used to get a fast and direct feedback from users in an early stage of the design process. Furthermore, they help to uncover relevant information about users and their work processes that should be supported [14].

Fast ways to create prototypes include the use of cardboards, paper, transparent paper, pens, and post-it notes. Literature on paper prototyping emphasizes, that creating a common understanding of an interface using a quickly drawn sketch positively influences the development process. In other words, users and developers often find it easier to understand a sketch, compared to discuss design options using a written document. Sketches or paper prototypes show first ideas and build the basis for discussions of further iteration stages. Additionally, they serve as a thinking tool, which support content-based analysis [7]. First low-fidelity prototypes, by means of sketches, are later transformed into more dynamic and flexible paper prototypes to encourage users to test, evaluate and change design ideas. This modular framework includes paper-based prototype-elements and placeholders. The elements can be fixed or dynamic to be placed individually during the design decision process. Placeholders are use to be drawn on and function as additional new elements of the interface. Moreover, alterable paper prototypes inspire a productive design discussion. With this modular framework [7] the developer-team gains valuable user feedback during the development process. This user feedback and the expression of user's ideas feed into subsequent stages of the interface design process. For the developer-team, the evolving discussion concerning the paper-based artifact and feedback on existing designs is especially fruitful to improve the interface design [15]. However, the lifecycle of a paper prototype is usually short. As soon as they served their purpose, they are generally thrown away or kept for emotional reasons [14]. In sum, paper prototyping is used for horizontal prototyping and mostly remain low-fidelity prototypes to show the overall functionality.

To extend the applicability of paper prototyping, i.e. to also discuss specific functions in great detail, the combination and rearrangement of previously printed interface elements is one efficient solution. The used materials are still paper and cardboard. Pen and glue are used for additional extensions during the prototyping process.

DOW introduces the possibility to create multiple prototypes [15]. He demonstrates that sharing multiple prototypes produce better results than dedicating the time to only one design idea. The final layout is inspired by all other designs. Group members will be more open to criticize, explore opportunities and discuss all prototypes. It's more likely, that there are less emotional bonds to any one idea [15]. "Collaborating on paper prototypes not only increases participation in the design process, but also improves communication among team members and increases the likelihood that the final design solution will be well accepted" [14]. However, it seems to be adequate to use multiple prototypes only in situations in which a creative mode of interaction among users and developers is really wanted. 


\subsection{Advantages and Disadvantages of Paper Prototyping}

Paper prototyping is known as a fast way to mock up an interface and to get fast and direct feedback from the user [16]. Therefore it is used in the early development in the field of interface design. SNYDER quotes that due to paper prototyping the communication in a multidisciplinary team, the user as a part of the team, is easier. Furthermore it encourages creativity from the developer team and the user as well. With paper as material for prototypes it is possible to produce different design solutions and present a variety of interface mock ups. Therefore paper prototyping is used for an effective decision finding process [15]. By examining the alternative designs, it is possible to choose the most efficient one [14].

One of the disadvantages is that there will be no code produced. A paper prototype cannot refer to all problems of the interface. Especially the fast and easy way of an interaction is hard to recreate. Furthermore the user might get a wrong impression of the interface [16].

\section{$3 \quad$ Paper Prototyping and Interactive Visualizations}

To study the main research question an in-depth case study method is applied [17], analyzing an interface design project at BMW. BMW is the world leading car manufacturer in the premium segment. BMW has to challenge existing processes, methods and models to maintain this market position. To address this challenge, BMW always searches for new ways to do things even better. Paper prototyping is seen as an innovative approach to create better results faster.

More specifically, paper prototyping is embedded in an interface design process of an HTML5 web interface that enables a new business process. Numerous experts concerning the design of the business process, technological experts, and operation experts contributed to the success of the project. This process addresses stages such as requirements felicitation, prototyping, and refinement in iterative cycles. In other words, it follows the idea of design science [18], while putting a rapid form of prototyping, in this case paper prototyping [19], at the center of design efforts.

To consolidate the framework of design science the user centered design [20] is applied. In four iterative cycles an interactive visualization is developed by putting users into the center of the design process without confronting them with technical details. Hence, our interdisciplinary team used paper prototyping throughout all cycles. This method allowed us to get the same understanding of the visualization and build a common language through images. Interaction possibilities were also simulated using paper prototypes. In detail, the following cycles with the respective stages of task analysis, design, prototype and test [21] were part of the development process:

\footnotetext{
$1^{\text {st }}$ cycle: Developing rough sketches

$2^{\text {nd }}$ cycle: Elaborate the visualization concept

$3^{\text {rd }}$ cycle: Enhance the visualization with an interaction concept

$4^{\text {th }}$ cycle: Implementing a HTML5 interactive visualization
} 
First cycle, we started by analyzing the task of the visualization. In the presented case we focused on developing a visualization for a new business process which shows complex information such as dependencies and a high number of dimensions. To prepare the paper prototyping the design phase aimed for a creative mood through inspiration (see fig. 3).

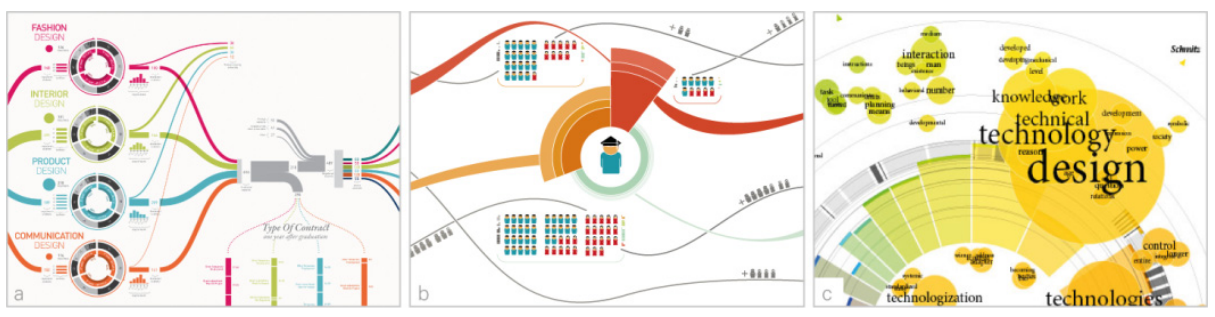

Fig. 3. Visualization-concepts as inspiration: (a) structure and efficiency of a School of Design [22], (b) Friendship-visualization [23], (c) „Total Interaction- “ connections between the book's content [24]

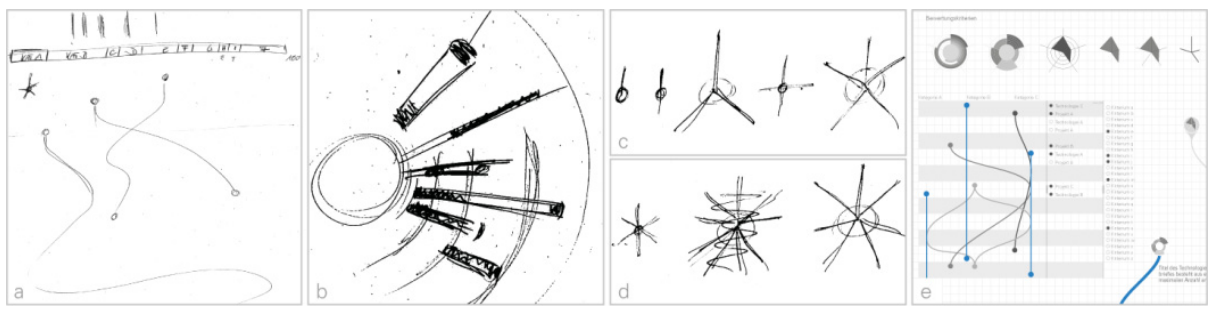

Fig. 4. Paper prototyping, first iteration: (a-d) rough sketches of ideas and (e) first iterations of the sketches on the PC

On this basis, first ideas were brainstormed and put into rough sketches (see fig. 4), which were reviewed by experts during the test phase.

Second cycle, with the most favored design idea the visualization concept was elaborated. To prepare the paper prototyping we identified the essential data and design elements and developed their first graphical representation (see circles in fig. 5). Putting all elements together as a paper prototype allowed the team and the users to discuss the visualization concept in detail and enhance it. Therefore we placed the single elements on a drawing area (see fig. 5), rearranged them and brought new design ideas into the scene. By drawing on existing elements, creating new sketches or printing new designs the graphical representations were designed and tested in an intuitive and rapid manner.

Third cycle, the paper prototype of the visualization allowed us to develop an interaction concept easily. In line with BEAUDOUIN-LAFON [14] we distinguished that the offline prototype made it comfortable for the team and the users to try different activities. The paper prototype facilitates to analyze, design, prototype and test diverse scenarios with varying interaction concepts and impact on the visualization. Simulating different tasks increased the comprehension of the user's needs. 


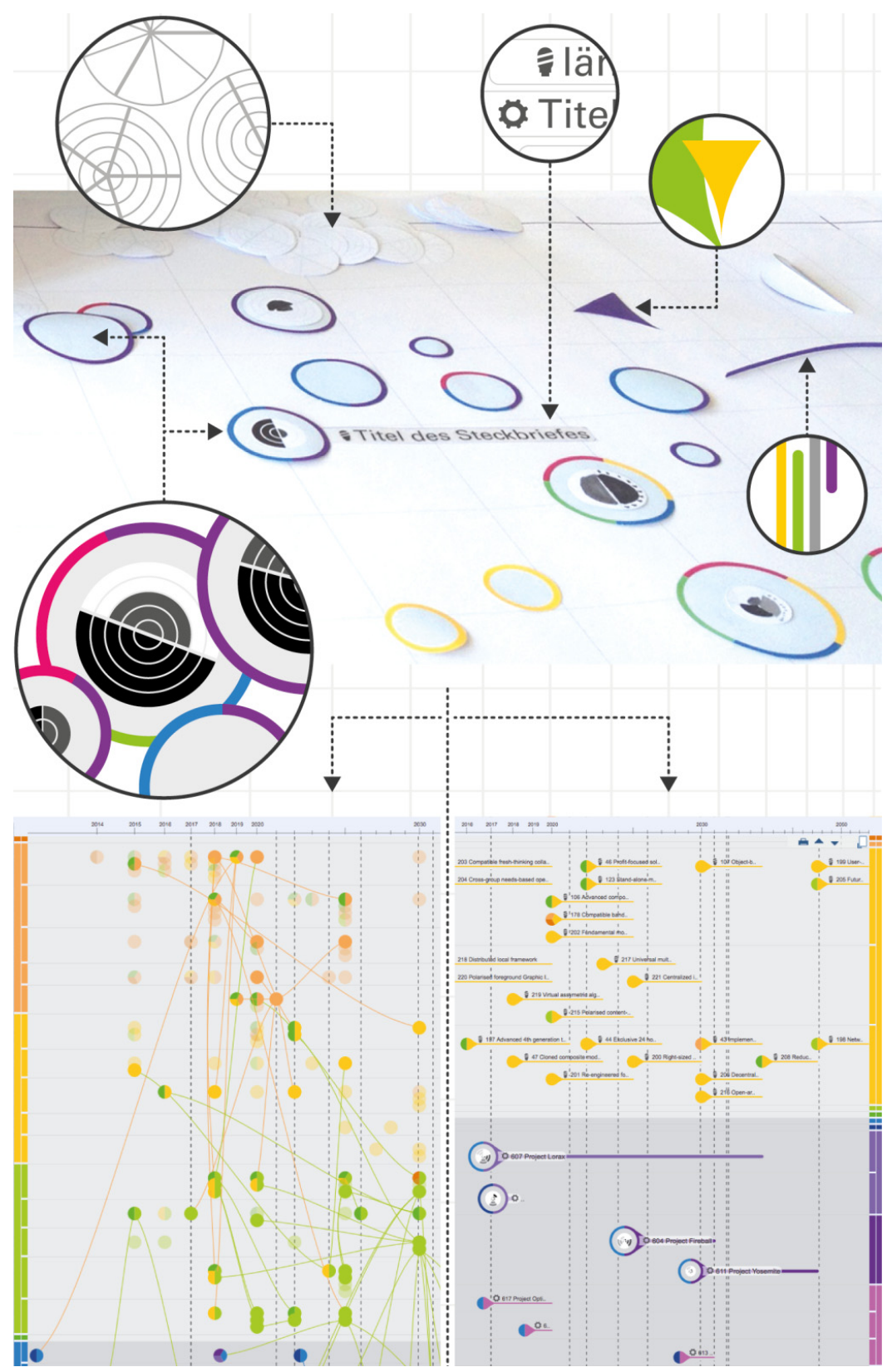

Fig. 5. Paper prototyping for interactive visualizations: Paper prototyping used for a design decision process (top) and results of the prototyping process implemented to the interface (bottom)

Fourth cycle, based on the developed concept we implemented an interactive visualization with HTML5 (see bottom part of fig. 5). The prior iterations helped to 
generate a common ground on requirements, so that the design, implementation and test in the fourth cycle were focused on technical details. Indeed, for further needs of clarification the paper prototype was used. That allowed the interdisciplinary team to discuss specifications in deep and overcome the issue of special terminology.

\section{Outcome}

Altogether, the results of the interface design project applying the above described paper prototyping flow seem to be very promising. Especially in circumstances characterized by (i) uncertainty concerning requirements, (ii) high time pressure and low availability among the experts able to formulate requirements, and (iii) innovativeness of the conceived software i.e. the most difficult situation to execute a software project, paper prototyping unfolds its full potential.

In our case study five advantages become apparent to the research team. First, the enjoyment aspect of working with a paper prototype is a very important facet we found in this study. Developers and users achieved a state of 'playing' with the paper material, the colored circles and lines, the net pieces, and texts. Watching children playing Lego may come closest to this state we observed. In this state, users and developers are working very concentrated on the prototype, make jokes here and there but always work as a whole group. In sum, enjoyment enables to set up a very concentrated atmosphere among a diverse set of participants, also under conditions of time pressure. This aspect of paper prototyping is widely ignored in current literature.

Second, a high degree of creativity among participants is achieved. The following situation explains this aspect. During the development process using the paper prototype, the group was confronted with a decision to choose one of two or more alternatives. However, the group was more often not deciding in favor of one alternative, but was searching for a completely new interpretation which would take the best of both (or more) prior alternatives. In sum, highly creative solutions were found for problems that seemed to be unsolvable. This finding supports SNYDER's list of advantages of paper prototyping, and adds some detail.

Third, the richness of the given feedback helped to reduce the necessary number of iterations drastically, while at the same time increasing the quality of the user interface and therefore the developed software. Participants use the paper prototype not only to visualize the conceived solution, but also reflected their daily experience upon the prototype. In other words, especially users were able to simulate or to envision the usage of the software in their work environment and to explicate their thoughts. Hence, developers are able to empathize the user's view based on very detailed and high quantity of feedback given. Especially this aspect contradicts with disadvantages often discussed.

Fourth, acceptance of the software in later stages increased drastically. This finding is especially remarkable as the development team was not able to include by far not all wishes or requirements. The acceptance of the software is mostly based on the feeling of all participants to be a part of the development team, which is in part true. So, introducing the new software is achieved more fluent. 
Fifth, the time needed to occupy experts and to develop the software was reduced significantly. All the prior mentioned aspects result in drastic efficiency gains. Less meetings, instant solutions of wicked problems, good understanding of requirements and highly accepted solutions are the outcomes of the paper prototyping project in this study.

Besides these positive outcomes, we also found a backside of paper prototyping. First, getting participants into creative mode is a challenge. As long as participants do not touch the paper the positive effects may not unfold as described above. Instead, developers as well as users stick to a discussion and may get distracted. So getting participants into a mode in which they work pro-actively with the paper material is essential.

Second, developers who prepare the paper prototyping session may get stuck to a particular design. This finding seems to be natural as the developers spend a lot of time with preparing the materials and have the time to develop an own understanding of the software. This effect is tricky as the developers are often unaware about this process as the development of an own understanding happens unconsciously. To not fall into this trap as a developer the only way is to remain open minded and curious.

\section{Conclusion}

The major aim of this paper was to close the gap between advantages of agile software development methods and challenges raised by a distributed workforce, such as existing at BMW. For this study a single in-depth case study approach was applied, following a design science paradigm. In greater detail, the method of paper prototyping was applied in four cycles of interface design of an innovative business application to be implemented at BMW.

The results of the study show that paper prototyping is superior to other methods of software development, mainly by realizing five main advantages. Two disadvantages were also identified. On the one hand, advantages include (1) enjoyment of all participants along the design process, (2) a high level of creativity that is unleashed, (3) high richness of feedback given by users, (4) the acceptance of the developed design in later stages of development, and (5) drastic reduction in development time. On the other hand, disadvantages include (1) the challenge to get all participants in a creative mode, and (2) developers being stuck to their previously developed design propositions. Altogether advantages overweight the disadvantages by far, not only on quantifiable measures such as development time and coding quality etc. but also on more subjective measures such as enjoying the development process. In sum, we were able to show how paper prototyping may be applied in distributed development teams in a big organization and the positive and negative effects of paper prototyping.

Future work could shed more light on the four development stages we applied. Specifically, it should be tested if the stages we identified hold also in other contexts or may be distinct to the in-depth case study at BMW. Furthermore, we also found that not every participant (developer and user) was able to give input in every stage. E.g., developers were more involved in early stages of drawing sketches while users 
involved more in working with the elements of the later paper prototype. Hence, future work could address this issue by identifying roles the different stakeholders play and exploring how these different roles may be included most effectively.

Summarizing this study, we were able to show how paper prototyping in an interface design process may work within a big company, and how paper prototyping effects the development process altogether. Avenues for future research are also shown.

\section{References}

1. Blackburn, J.D., Scudder, G.D., Van Wassenhove, L.N.: Improving Speed and Productivity of Software Development: A Global Survey of Software Developers. IEEE Transactions on Software Engineering 22(12), 875-885 (1996)

2. Dag, J.N., Gervasi, V., Brinkkemper, S., Regnell, B.: Speeding up Requirements Management in a Product Software Company: Linking Customer Wishes to Product Requirements through Linguistic Engineering. In: 12th IEEE International Requirements Engineering Conference (RE 2004), pp. 283-294. IEEE Computer Society (2004)

3. Cohen, D., Lindvall, M., Costa, P.: Agile Software Development. DACS State-of-theArt/Practice Report, Fraunhofer Center Maryland (2003)

4. Highsmith, J.: Extreme programming. White Paper, Arlington (2000)

5. Beck, K., Beedle, M., Bennekum, A., van Cockburn, A., Cunningham, W., Fowler, M., Grenning, J., Highsmith, J., Hunt, A., Jeffries, R., Kern, J., Marick, B., Martin, R.C., Mellor, S., Schwaber, K., Sutherland, J., Thomas, D.: Manifesto for Agile Software Development, http: / / agilemanifesto.org

6. Ramasubbu, N., Balan, R.K.: Globally Distributed Software Development Project Performance: An Empirical Analysis. In: 6th Joint Meeting of the European Software Engineering Conference and the ACM SIGSOFT Symposium on the Foundations of Software Engineering (ESEC-FSE 2007), pp. 125-134. ACM Press, New York (2007)

7. Knöfel, A., Koalick, G., Lapczyna, E., Groh, R.: Pimp your prototype. In: 5th International Scientific Conference "Printing Future Days 2013", pp. 131-136. VWB - Verlag für Wissenschaft und Bildung, Berlin (2013)

8. Nielsen, J.: Usability Engineering. Academic Press, London (1994)

9. Sears, A., Jacko, J.A.: The Human-Computer Interaction Handbook: Fundamentals, Evolving Technologies and Emerging Applications. Lawrence Erlbaum Associates, Taylor \& Francis Group, New York, London (2012)

10. Moggridge, B.: Designing Interactions. The MIT Press, Cambridge (2007)

11. Chair of Media-Design: Werkstatt, http://werkstatt.inf .tu-dresden.de

12. Rudd, J., Stern, K., Isensee, S.: Low vs. High-Fidelity Prototyping Debate. Interactions 3(1), 76-85 (1996)

13. Rettig, M.: Prototyping for Tiny Fingers. Communications of the ACM 37(4), 21-27 (1994)

14. Beaudouin-Lafon, M., Mackay, W.E.: Prototyping Tools and Techniques. In: Sears, A., Jacko, J.A. (eds.) The Human-Computer Interaction Handbook: Fundamentals, Evolving Technologies and Emerging Applications, pp. 1017-1039. Lawrence Erlbaum Associates, Taylor \& Francis Group, New York, London (2012)

15. Dow, S.: How prototyping practices affect design results. Interactions 18(3), 54-59 (2011)

16. Snyder, C.: Paper Prototyping: The Fast and Easy Way to Design and Refine User Interfaces. Morgan Kaufmann Publishers, San Francisco (2003) 
17. Yin, R.K.: Case Study Research: Design and Methods. SAGE Publications, Los Angeles (2009)

18. Hevner, A., Chatterjee, S.: Design Research in Information Systems. Springer Science+Business Media, New York (2010)

19. Sefelin, R., Tscheligi, M., Giller, V.: Paper Prototyping - What is it good for? A Comparison of Paper- and Computer-based Low-fidelity Prototyping. In: Extended Abstracts on Human Factors in Computing Systems (CHI 2003), pp. 778-779. ACM Press, New York (2003)

20. Norman, D.: Design Principles for Human-Computer Interfaces. In: Conference on Human Factors in Computing Systems (CHI 1983), pp. 1-10. ACM, New York (1983)

21. Tory, M., Möller, T.: Human Factors in Visualization Research. IEEE Transactions on Visualization and Computer Graphics 10(1), 72-84 (2004)

22. De Donno, S.: Visualizing the School of Design, http: / /www.densitydesign.org/2013/04/visualizing-theschool-of-design/

23. Eschrich, B., Leitner, H.: Freundschaft, http://issuu.com/brino/docs/freundschaft

24. Rembold, M., Späth, J.: http: //www. munterbund. de, http://www. munterbund. de 